\title{
Mu2e Muon Beam Optimization
}

\author{
Helenka Casler* on behalf of the Mu2e Collaboration \\ CUNY York College, 94 - 20 Guy R. Brewer Blvd. Jamaica, NY 11451, USA and CUNY \\ Graduate Center, 365 Fifth Avenue, New York, NY 10016, USA \\ E-mail: hcasleregc.cuny.edu
}

\begin{abstract}
Mu2e is a new experiment under construction at Fermilab, which will search for coherent neutrinoless conversion of muons to electrons. In order to reach its projected single-event sensitivity of $3 \times 10^{-17}$, Mu2e will create the most intense muon beam ever developed, with $10^{10}$ muons per second stopping in the stopping target. Optimization of this muon beam for Mu2e will be discussed.
\end{abstract}

The 21st international workshop on neutrinos from accelerators (NuFact2019)

August 26 - August 31, 2019

Daegu, Korea

\footnotetext{
*Speaker.

†http://mu2e.fnal.gov

¥Collaboration list: https://mu2e.fnal.gov/mu2e_collaboration_list.shtml
} 


\section{Introduction}

The Mu2e experiment at Fermilab will search for charged lepton flavor violation (CLFV) with a single-event sensitivity of $3 \times 10^{-17}$. Currently under construction, Mu2e will search for the interaction $\mu N \rightarrow e N$, or the neutrinoless conversion of a muon to an electron in the field of an atomic nucleus. This interaction has never been observed experimentally, but is allowed to occur within the Standard Model by coupling neutrino oscillations with loops. However, the branching ratios for these interactions rely on the ratio of the squares of differences between the neutrino masses to the $\mathrm{W}$ boson mass, giving them extremely tiny values

$$
\mathrm{BR}(\mu \rightarrow e)=\frac{3 \alpha}{32 \pi}\left|\sum_{k=2,3} U_{\mu k}^{*} U_{e k} \frac{\Delta m_{1 k}^{2}}{M_{W}^{2}}\right|^{2} \sim 10^{-54} .
$$

Here, $U$ refers to the PMNS matrix. It is not possible for any experiment to measure a branching ratio this small. Any experiment which does see $\mu N \rightarrow e N$, then, has found direct evidence of physics beyond the Standard Model.

Searches for CLFV constitute a thriving ecosystem of physics experiments. In addition to LHC searches in meson and tau channels, there are a number of muon-focused efforts worldwide, including several in this workshop. Besides Mu2e, the search for neutrinoless $\mu N \rightarrow e N$ includes the DeeMe [1] and COMET [2] experiments at JPARC, also currently under construction. Mu3e, under construction at PSI, is searching for $\mu^{+} \rightarrow e^{+} e^{+} e^{-}$[3], while MEG (also at PSI) was able to push the upper limit for $\mu \rightarrow e \gamma$ to $4.2 \times 10^{-13}$ [4], and is about to begin collecting data after a major upgrade [5].

\subsection{Experimental Design}

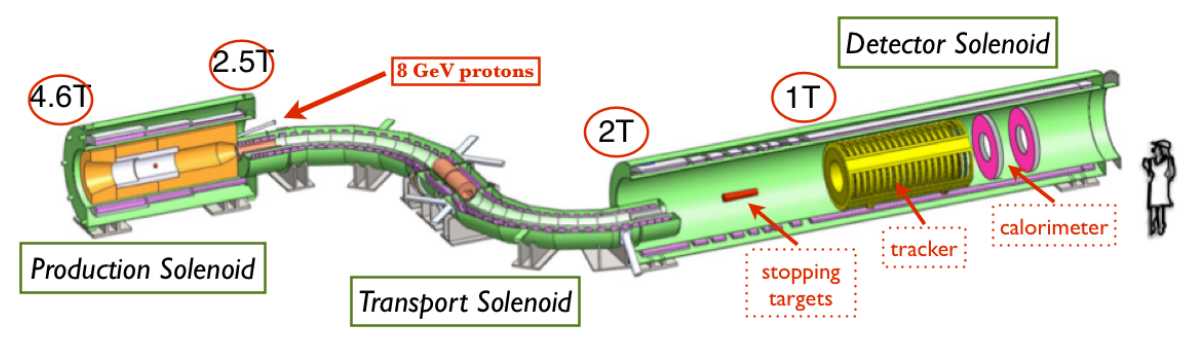

Figure 1: Layout of the Mu2e experiment. The solenoids are labeled, as are the stopping target and two of the detectors. Magnetic field values at certain points are circled in red.

The Mu2e experiment (Figure 1) is composed of three solenoids - the Production Solenoid (PS), Transport Solenoid (TS), and Detector Solenoid (DS). This design separates the production of muons from the observation of their decays, keeping a quiet environment in the DS. Muons are produced inside the production solenoid, by colliding the proton beam with the production target. This collision produces pions, which quickly decay to muons. The transport solenoid then directs the muon beam to the stopping target, which is located inside the detector solenoid. Once 
they reach the aluminum stopping target, the muons are slowed by Coulomb interactions until they reach thermal energies and are captured in orbit around an atomic nucleus. They quickly cascade to the $1 \mathrm{~s}$ state on a timescale $\left(10^{-13} \mathrm{~s}\right)$ which is significantly shorter than the muon lifetime in aluminum (864 ns). At this point, they will either be captured by the nucleus, undergo regular decay $(\mu \rightarrow e v v)$ in orbit, or they may convert to electrons. If a conversion occurs, the electron is ejected from the $\mathrm{Al}$ atom, and will spiral in the magnetic field of the DS to be detected by the tracker and calorimeter.

Mu2e must contend with several background sources, including cosmic rays, muon decay in orbit, antiprotons, radiative pion capture (RPC), and others [6]. The experimental design has been optimized to reduce these backgrounds to the fullest extent possible, resulting in less than one background event over the course of the entire experiment [6]. Of these background sources, RPC has the greatest implications for the muon beam structure. RPC refers to the nuclear capture of a charged pion from the PS in the aluminum target. Target nuclei that capture pions become excited and quickly release photons, which can then pair-produce an electron and positron. These electrons can become background events if they have energies close to the conversion electron energy from aluminum of $104.96 \mathrm{MeV}$. However, the lifetime of the charged pions is $26 \mathrm{~ns}$, placing a time limit on RPC events. This time limit allows us to avoid RPC backgrounds if we make use of a pulsed proton beam, as described in the next section.

For more details on the design of Mu2e and its current status, see the presentation by $\mathrm{R}$. Bonventre given at the Muon Physics (WG4) workshop at this conference. For details on Mu2e II, a planned upgrade to Mu2e, see the presentation by I. Oksuzian given at the same workshop.

\section{Beam Time Structure}

The muon beam time structure is driven by the requirement for low physics backgrounds and its effect on the proton beam time structure. When the proton beam hits the production target, some of the resulting pions will reach the DS. Once there, they may undergo radiative pion capture in the stopping target, as described in Section 1. We can avoid detecting these events by utilizing a pulsed proton beam time structure, and only attempting to find a signal long after the pions from the pulse would have decayed or annihilated on matter. On the other hand, we do not want to miss conversion electrons by closing our detection time window while there are still muons in orbit in the stopping target. This sets our proton beam time structure: the pulse separation must be greater than the muonic aluminum lifetime. "Stray" pions that enter the DS can still undergo RPC, so those must be kept to a minimum. Therefore, we require the extinction between pulses (ratio of out-of-pulse protons to in-pulse protons) to be below $10^{-10}$.

\subsection{Proton Beam Manipulations}

Fermilab's muon program is run with $8 \mathrm{GeV}$ protons. These $8 \mathrm{GeV}$ protons are injected into the Recycler Ring from the Booster, which is also shared with the neutrino program. The NOvA experiment requires $120 \mathrm{GeV}$ protons, so while the Main Injector is ramping up to $120 \mathrm{GeV}$, two $8 \mathrm{GeV}$ batches are injected into the Recycler, totalling about $8 \times 10^{12}$ protons. The Recycler 2.5 $\mathrm{MHz}$ RF system then rebunches the two Booster batches into eight Recycler bunches in $90 \mathrm{~ms}$. 
Once the rebunching is complete, each bunch is individually transferred to the Delivery Ring (DR), every $48.1 \mathrm{~ms}$, until all eight have been transferred.

After a bunch is injected into the DR, it is then slow-spilled into the Mu2e beamline via resonant extraction for delivery to the Mu2e target. The extraction process involves intentionally driving a $1 / 3$ integer resonance in the horizontal tune using the DR quadrupoles. Meanwhile, the DR sextupoles induce a controlled beam instability, causing the beam to grow in the horizontal plane. On each turn around the ring, an electrostatic septum peels off a microbunch and sends it to Mu2e. The orbital period of the DR is $1695 \mathrm{~ns}$ - this is about twice the muonic aluminum lifetime, making it almost the ideal time structure for Mu2e.

\subsection{Extinction System}

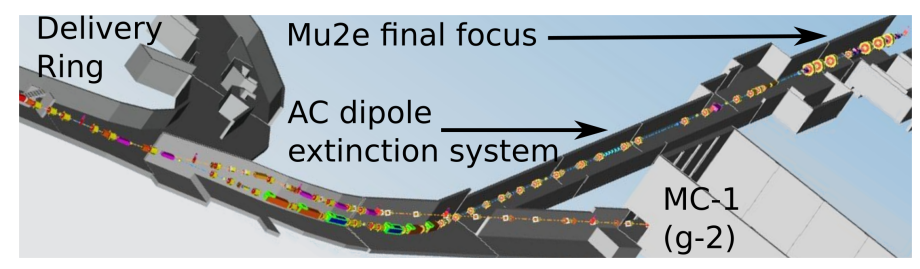

Figure 2: Location of the extinction system, between the exit from the Delivery Ring (left) and Mu2e.

Mu2e uses an AC dipole extinction system, located in the Mu2e beamline (Figure 2). The AC dipole system consists of a pair of resonant dipoles, which are excited on two harmonics: $300 \mathrm{kHz}$ and 4.5 MHz. The $300 \mathrm{kHz}$ frequency allows in-time beam to pass through on the nodes (for a pulse rate of $600 \mathrm{kHz}$ ), while deflecting out-of-time beam into a set of absorbing collimators. The 4.5 MHz harmonic is timed to reduce the magnet slewing during the passage of in-time beam.

In order to avoid deflecting out-of-time beam from outside the expected admittance into the transmission line, a system of halo and tail collimators are placed upstream of the AC dipole to precisely define the admittance. The AC dipole extinction system overall is expected to improve the extinction by a factor of $5 \times 10^{-8}$, from $2 \times 10^{-5}$ coming from the Delivery Ring to $10^{-12}$. Thus we can expect to exceed our extinction requirement by two orders of magnitude.

\section{Particle Selection}

Mu2e will be searching for monoenergetic electrons at $104.96 \mathrm{MeV}$. In order to avoid muddying this signal, we must prevent any other charged particles with approximately that energy from entering the tracker or calorimeter. Muons which enter the DS ought to be limited to energies significantly below $100 \mathrm{MeV}$, and all other particles ought to be prevented from entering the DS at all during the detection window. Selection of low-energy negative muons, to the exclusion of other particles, is carried out by the Transport Solenoid.

The Transport Solenoid (TS) has a unique S shape, consisting of two ninety-degree turns in opposite directions. This curved shape excludes neutral particles from the Production Solenoid from reaching the DS. The TS magnetic field varies between 2 and $2.5 \mathrm{~T}$, and the field lines are shaped to follow the curve. Charged particles spiral in the magnetic field, guided by the field lines. 
The shape of the TS is such that particles whose momenta exceed $80 \mathrm{MeV}$ will not make it around the bend. The toroidal field in the TS also aids in charge selection. In the upstream curve, negative particles drift upward, while positive ones drift down. In the center of the TS is a pair of collimators which can be rotated, to select only those particles in the upper or lower portion of the solenoid. In the downstream section, the solenoid curves in the opposite direction, causing the selected particles to drift back to the center.

While the shape of the TS field allows us to screen out neutral particles and fast particles, this alone is not enough to ensure a clean muon beam. The rest is taken care of by antiproton windows. There are two: one at the entrance to the TS from the Production Solenoid, and the other in the center, between the center collimators. These thin windows block antiprotons and radioactive ions from the production target. The remaining particles that can make it through the TS are negative muons, a few slow electrons which can easily be distinguished from signal electrons because of their lower energy, and slow pions, which are already excluded by pulse and detection timing.

\section{Acknowledgments}

We are grateful for the vital contributions of the Fermilab staff and the technical staff of the participating institutions. This work was supported by the US Department of Energy; the Italian Istituto Nazionale di Fisica Nucleare; the Science and Technology Facilities Council, UK; the Ministry of Education and Science of the Russian Federation; the US National Science Foundation; the Thousand Talents Plan of China; the Helmholtz Association of Germany; and the EU Horizon 2020 Research and Innovation Program under the Marie Sklodowska-Curie Grant Agreement No.690385. Fermilab is operated by Fermi Research Alliance, LLC under Contract No. De-AC0207CH11359 with the US Department of Energy, Office of Science, Office of High Energy Physics. The United States Government retains, and the publisher, by accepting the article for publication, acknowledges, a non-exclusive, paid-up, irrevocable, world-wide license to publish or reproduce the published form of this manuscript, or allow others to do so, for United States Government purposes.

\section{References}

[1] DeeMe collaboration, M. Aoki, A new idea for an experimental search for nu-e conversion, in proceedings of ICHEP 2010, POS ( ICHEP 2010) 279 (2010).

[2] COMET Collaboration, Conceptual design report for experimental search for lepton flavor violating mu- - e-conversion at sensitivity of $10^{16}$ with a slow-extracted bunched proton beam (COMET), KEK-2009-10 (2009).

[3] Mu3e collaboration, N. Berger, The Mu3e Experiment, Nucl. Phys. Proc. Suppl. 248-250 (2014) 35.

[4] MEG collaboration, Search for the lepton flavour violating decay $\mu^{+} \rightarrow e^{+} \gamma$ with the full dataset of the MEG experiment, Eur. Phys. J. C 76 (2016) 434, [1605.05081].

[5] MEG II collaboration, A. M. Baldini, Status of the MEG II experiment at PSI. J. Phys.: Conf. Ser. 1137 (2019) 012028.

[6] Mu2e collaboration, Mu2e Technical Design Report, FERMILAB-TM-2594, FERMILAB-DESIGN-2014-01 (2014), [1501.05241]. 